\title{
T cell activation and anti-tumor efficacy of anti- LAG-3 antibodies is independent of LAG-3 - MHCII blocking capacity
}

\author{
Saso Cemerski ${ }^{1 *}$, Shuxia Zhao ${ }^{1}$, Melissa Chenard ${ }^{1}$, Jason Laskey ${ }^{1}$, Long Cui ${ }^{1}$, Rinkan Shukla ${ }^{2}$, Brian Haines ${ }^{1}$, \\ Edward Hsieh², Maribel Beaumont ${ }^{2}$, Jeanine Mattson², Wendy Blumenschein ${ }^{2}$, Heather Hirsch', \\ Laurence Fayadat-Dilman², Linda Liang ${ }^{2}$, Rene De Waal Malefyt ${ }^{2}$
}

From 30th Annual Meeting and Associated Programs of the Society for Immunotherapy of Cancer (SITC 2015) National Harbor, MD, USA. 4-8 November 2015

LAG-3 has been shown to act as an inhibitory molecule involved in the regulation of $\mathrm{T}$ cell activation, proliferation and homeostasis. Exhausted T cell populations that evolve in the tumor microenvironment or during chronic viral infections show coordinated expression of LAG-3 and PD1. LAG-3 is structurally related to CD4 and binds to MHCII. Anti-LAG-3 antibodies have demonstrated preclinical efficacy in several disease models in particular when combined with anti-PD-1 antibodies. Studies have proposed that LAG-3 blockade is efficacious in both CD4 ${ }^{+}$ and $\mathrm{CD} 8^{+} \mathrm{T}$ cells despite the lack of significant MHCII levels on $\mathrm{CD} 8^{+} \mathrm{T}$ cell. In the present study, we evaluated if anti-LAG-3 efficacy is dependent on the ability of the antibody to inhibit the binding of LAG-3 to MHCII.

We have compared in a series of in vitro assays the biological activity of two distinct anti-mouse LAG-3 antibodies: C9B7W which does not block the LAG-3 - MHCII interaction and an in-house generated antibody 28G10 that strongly blocks the interaction between LAG-3 and MHCII. Biophysical characterization revealed that C9B7W and 28G10 recognize distinct epitopes on LAG-3, therefore explaining the difference in LAG3-MHCII interruption. However, no differences were observed in $\mathrm{T}$ cell activation assays between the two antibodies using TCR transgenic $\mathrm{CD} 4^{+} \mathrm{T}$ cells. In addition, their ability to synergize with an anti-PD-1 antibody was also comparable.

To understand if the overall enhancement in $\mathrm{CD} 4^{+} \mathrm{T}$ cell activation by C9B7W and 28G10 was achieved through different mechanisms, we profiled gene expression in T cells stimulated in the presence of anti-LAG-3 antibodies. No significant difference was found between the two antibodies. Consistent with this observation, we did not see an additive effect of C9B7W and 28G10 when used together in vitro. Neither antibody demonstrated a significant effect on the activity of TCR transgenic $\mathrm{CD} 8^{+} \mathrm{T}$ cells in in vitro functional assays.

Furthermore, the two antibodies demonstrated comparable anti-tumor efficacy in in vivo syngeneic tumor models when dosed in combination with anti-PD-1.

In conclusion, our studies demonstrate that the activity of LAG-3-targeting antibodies is not associated with their ability to disrupt LAG-3-MHCII interaction. This would suggest that anti-LAG-3 antibodies should enhance both $\mathrm{CD}^{+}$and $\mathrm{CD}^{+}{ }^{-} \mathrm{T}$ cell function. Case in contrast, our in vitro data demonstrates that LAG-3 targeting augments the activation of $\mathrm{CD} 4^{+} \mathrm{T}$ cells significantly more than $\mathrm{CD} 8^{+}$ $\mathrm{T}$ cells.

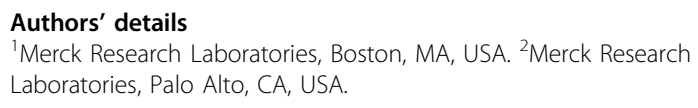

Published: 4 November 2015

doi:10.1186/2051-1426-3-S2-P183

Cite this article as: Cemerski et al:: T cell activation and anti-tumor

efficacy of anti-LAG-3 antibodies is independent of LAG-3 - MHCI

blocking capacity. Journal for ImmunoTherapy of Cancer 2015 3(Suppl 2):P183.

${ }^{1}$ Merck Research Laboratories, Boston, MA, USA

Full list of author information is available at the end of the article 